\title{
AN EFFICIENT SEGMENTATION METHOD FOR MILK SOMATIC CELL IMAGES
}

\author{
Heru Xue ${ }^{1, *}$, Shuoshi $\mathrm{Ma}^{2}$, Xichun Pei ${ }^{1}$ \\ ${ }^{1}$ College of Computer and Information Engineering, Inner Mongolia Agricultural University, \\ Hohhot, China, 010018 \\ ${ }^{2}$ College of Machinery and Electrical Engineering, Inner Mongolia Agricultural University, \\ Hohhot, China, 010018 \\ * Corresponding author, Address: College of Computer and Information Engineering, Inner \\ Mongolia Agricultural University, 306 Zhaowuda Road, Hohhot, China, 010018, P. R. \\ China, Tel: +86-471-4309237, Fax: +86-471-4306865, Email: xuehr@imau.edu.cn
}

Abstract: The accurate segmentation of milk somatic cells in microscope images may contribute to development of a successful system that automatically analyzes, detects and counts cells in microscope images. We present a method for milk somatic cell Segmentation. Our approach is based on segmentation of subsets of bands using mathematical morphology followed by the fusion of the resulting segmentation "channels". For color images, the band subsets are chosen as RG, RB and GB pairs. The segmentation in $2 \mathrm{D}$ color spaces is obtained using the watershed algorithm. These $2 \mathrm{D}$ segmentations are then combined to obtain a final result using a region split-and-merge process. Milk somatic cell images are segmented, and background, nucleus and cytoplasm can be extracted correctly. The most important feature of this method is the improved performance.

Keywords: milk somatic cell, color image segmentation, mathematical morphology, image fusion

\section{INTRODUCTION}

Most Color image segmentation is basically a 3D image histogram clustering. Many methods for color image segmentation on 3D histogram have been developed so far. However, clustering a 3D histogram can be

Xue, H., Ma, S. and Pei, X., 2008, in IFIP International Federation for Information Processing, Volume 259; Computer and Computing Technologies in Agriculture, Vol. 2; Daoliang Li; (Boston: Springer), pp. 1253-1258. 
expensive because of huge amount of data involved. One remedy is to project the 3D color space into a lower dimensional space such as $2 \mathrm{D}$ (Kurugollu et al., 2001) or even 1D space (Cheng et al., 2000). In this paper, the method we present is partly based on the 2D morphological clustering method (Xue et al., 2003).

A typical milk smear consists of somatic cell, milk creaminess, lactoproteid, debris and so on. The goal of segmentation is to locate the milk somatic cells and to mark their nucleus and cytoplasm regions. Numerous methods have been proposed for digital cell images of peripheral blood (Comaniciu et al., 1999).

The above cell segmentations need to be accomplished in 3D space. Hence they have poor performance, especially in running time and memory consumption. In this paper, we present an improved approach for milk somatic cell color image segmentation.

\section{SEGMENTATION SCHEME}

\subsection{Segmentation in 2D color spaces}

Fig. 1 shows the procedure of the proposed segmentation scheme. The first step is to apply the morphological segmentation process (Géraud et al., 2001).

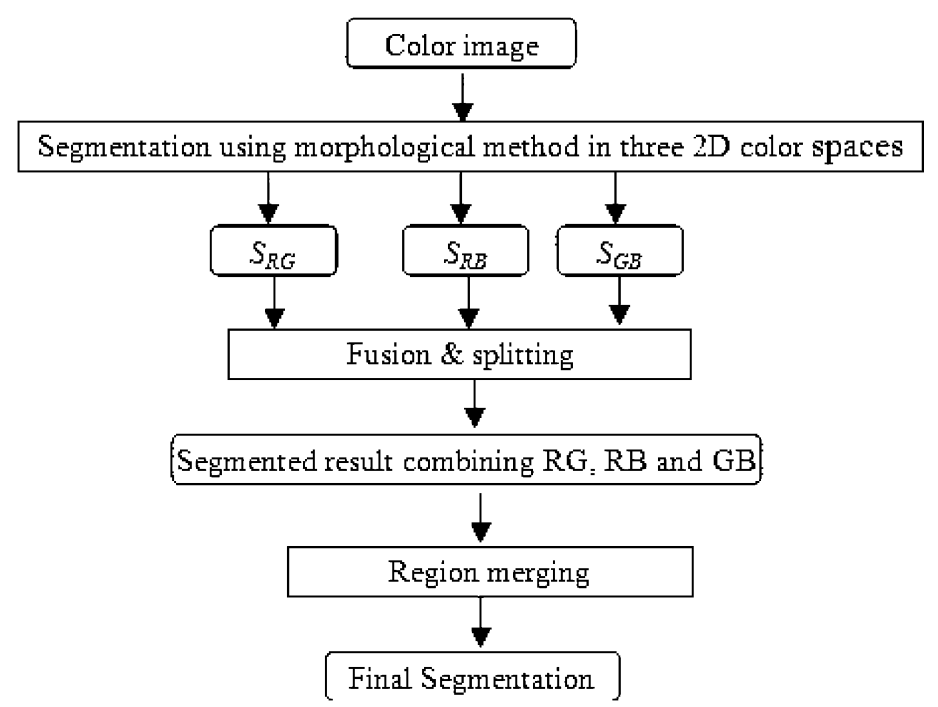

Fig. 1. The flowchart of the proposed approach 
Instead of computing a 3D histogram in the 3D RGB space, we compute these 2D histograms, respectively in the 2D color spaces RG, RB, and GB. For instance, $H_{R G}^{(0)}$ is a $2 \mathrm{D}$ histogram of input image $I$ which is only based on the red and green components.

From the three 2D histograms $H_{R G}^{(0)}, H_{R B}^{(0)}$, and $H_{G B}^{(0)}$, we output these segmented images $S_{R G}, S_{R B}$, and $S_{G B}$ using watershed algorithm (Géraud et al., 2001).

\subsection{Fusion of segmentation results}

None of these three 2D segmentations is good enough to be the final resulting segmentation of the original color image. So a fusion process of segmentation results is required.

\subsubsection{Fuzzy matching degree between two classes}

Let us symbolize by $T$ a $2 \mathrm{D}$ projection ( $T$ can be RG, RB, or GB), $l$ being a class label, we denote by $S_{T}^{(l)}$, the set of points assigned to class $l$ in the segmentation $S_{T}$. In other words:

$$
S_{T}^{(l)}=\left\{\mathrm{pl} S_{T}(p)=c_{T}^{l_{T}(p)}\right\}
$$

A partial similarity degree between both corresponding point sets is defined as:

$$
\mu_{T_{1} \rightarrow T_{2}}^{\left(l_{1}, l_{2}\right)}=\frac{\operatorname{card}\left(S_{T_{1}}{ }^{\left(l_{1}\right)} \cap S_{T_{2}}{ }^{\left(l_{2}\right)}\right)}{\operatorname{card}\left(S_{T_{1}}{ }^{\left(l_{1}\right)}\right)}
$$

Then, we get a fuzzy matching degree:

$$
\mu_{T_{1}, T_{2}}^{\left(l_{1}, l_{2}\right)}=\mu_{T_{1} \rightarrow T_{2}}^{\left(l_{1}, l_{2}\right)} \oplus \mu_{T_{2} \rightarrow T_{1}}^{\left(l_{1}, l_{2}\right)}
$$

where: $\oplus$ can be any fuzzy T-norm operator. Here, we use "max" operator.

\subsubsection{Region splitting}

Given a criterion $m \in(0,1)$, if $\mu_{T_{1}, T_{2}}^{\left(l_{1}, l_{2}\right)}>m$, we split set $S_{T_{1}}^{\left(l_{1}\right)}$ into two subsets $S_{T_{1}}^{\left(l_{1}\right)}-S_{T_{1}}^{\left(l_{1}\right)} \cap S_{T_{2}}^{\left(l_{2}\right)}$ and $S_{T_{1}}^{\left(l_{1}\right)} \cap S_{T_{2}}^{\left(l_{2}\right)}$.

The splitting process is iterative. For instance, we can take the order as illustrated in Fig. 2 in the case of RGB images. 


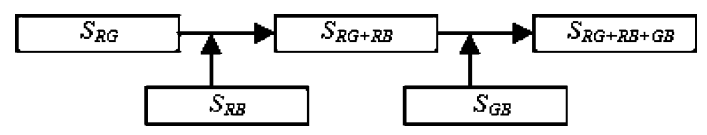

Fig. 2. Diagram of splitting process

The splitting process, while combining information from these classifications, separates nearly all classes that overlap in 2D color spaces.

\subsubsection{Region merging}

For input RGB image $I$, the result after the splitting process is an oversegmentation $S_{R G+R B+G B}$ of $I$. Therefore, a region merging is necessary. In the merging algorithm, the regions with the smallest color distance are first merged.

Here, we perform the algorithm in CIE $\mathrm{L}^{*} \mathrm{a}{ }^{*} \mathrm{~b}^{*}$ color space which has more uniform perceptual properties than other spaces (Cheng et al., 2000).

\section{MILK SOMATIC CELL IMAGE SEGMENTATION}

Large numbers of milk somatic cell images have been processed by our segmentation scheme. Fig. 3 shows the segmentation results of an image of a milk smear.

As can be shown, the background, cytoplasm and nucleus are correctly separated, even while the images don't have very good contrast between the background and the cytoplasm of the cell images. We utilize the fact that the nucleus has minimum gray value (g) for locating the milk somatic cells,

Where:

$$
\mathrm{g}=\frac{R+G+B}{3}
$$

Where R, G and B are the center of each class.

We have employed a large variety of milk somatic cell color images in our experiments. Some results of the proposed approach are shown in Fig. 3. The corresponding segmentation method using automatic morphological approach in 3D space (Géraud et al., 2001) is also tested for the comparison between the 3D method and our 2D method. The 3D method is based on 3D histogram and no fusion is needed. The experiments show that the somatic cell segmentations using the two methods are both correct. Furthermore, the execution time for the algorithm based on $2 \mathrm{D}$ space is less than that in $3 \mathrm{D}$ space. 


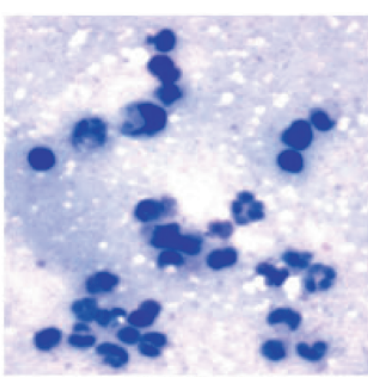

(a) Original

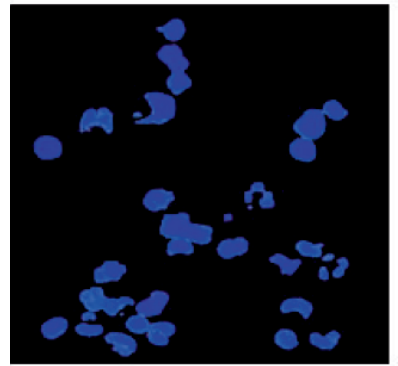

(c) Nucleus

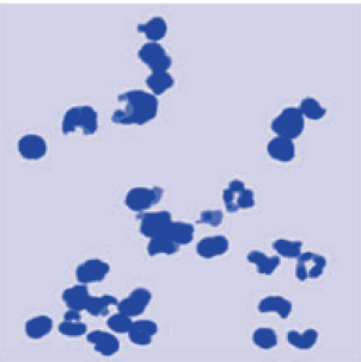

(b) Segmented images (3 classes)

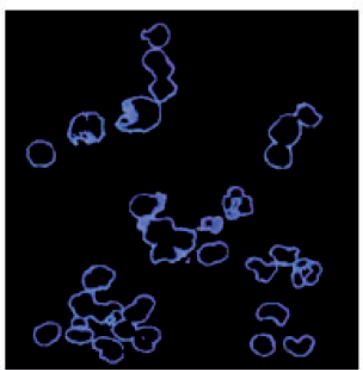

(d) Cytoplasm

Fig. 3. Segmentation results of milk somatic cell images

Some experimental results for the comparative performance between the 2D method proposed in this paper and the 3D method proposed by Geraud (2001), are listed in Table 1.

The experimental results indicate that both results of the 3D method and the ones obtained from the 2D method are correct. However the latter is more efficient, particularly regarding running time.

Table 1. Comparison between 2D and 3D methods

\begin{tabular}{crc}
\hline \multirow{2}{*}{ Images } & \multicolumn{2}{c}{ Running time (s) } \\
\cline { 2 - 3 } & $3 \mathrm{D}$ & $2 \mathrm{D}$ \\
\hline 1 & 58.13 & 5.91 \\
2 & 83.10 & 6.09 \\
3 & 101.05 & 8.91 \\
4 & 78.43 & 11.67 \\
5 & 88.40 & 6.04 \\
6 & 63.83 & 5.42 \\
\hline
\end{tabular}

\section{CONCLUSION}

In this paper, an efficient somatic cell image segmentation method has been presented. The method is based on morphological classification in 2D color space and a fusion technique. Large mount of milk somatic cell images 
are segmented by our method and the results are correct. This approach requires no user-interaction or parameter tuning, and the processing speed is fast.

\section{REFERENCES}

Cheng H D, Sun Y. A hierarchical approach to color image segmentation using homogeneity, IEEE Trans. On Image Processing, 2000, 9(12): 2071-2082.

Comaniciu D, Meer P. Mean Shift Analysis and Applications, IEEE Int'l Conf. Comp. Vis., Kerkyra, Greece, 1999: 1197-1203.

Géraud T, Strub P Y, Darbon J. Color image segmentation based on automatic morphological clustering, in Proc. IEEE International Conference on Image Processing, 2001: 70-73.

Kurugollu F, Sankur B, Harmanci A E. Color image segmentation using histogram multithresholding and fusion, Journal of Image and Vision Computing, 2001, 19(13): 915-928.

Xue H, Pei X, Géraud T, Ma S. Color image segmentation using fuzzy sets and fusion, in Conference Proceedings of the Sixth International Conference on Electronic Measurement \& Instruments (ICEMI'2003), 2003: 72-75. 